\title{
Word Sense Disambiguation with Pattern Learning and Automatic Feature Selection
}

\author{
Rada F. MIHALCEA \\ Department of Computer Science \\ University of North Texas \\ Denton, TX, 76203-1366 \\ rada@cs.unt.edu
}

(Received November 1, 2001; Revised: March 14, 2002)

\begin{abstract}
This paper presents a novel approach for word sense disambiguation. The underlying algorithm has two main components: (1) pattern learning from available sense-tagged corpora (SemCor), from dictionary definitions (WordNet) and from a generated corpus (GenCor), and (2) instance based learning with automatic feature selection, when training data is available for a particular word. The ideas described in this paper were implemented in a system that achieved the best score during the SENSEVAL-2 evaluation exercise, for both English all words and English lexical sample tasks.
\end{abstract}

\section{Introduction}

Word sense disambiguation (WSD) does not need any more an introduction and particularly not in a special issue on WSD evaluation. It is well known that WSD constitutes one of the hardest problems in Natural Language Processing, yet is a necessary step in a large range of applications including machine translation, knowledge acquisition, coreference, information retrieval and others. This fact motivates a continuously increasing number of researchers to develop WSD systems and devote time for finding solutions to this challenging problem.

The system presented here was initially designed for the semantic disambiguation of all words in open text. The SEnseval competitions provided a good environment for supervised systems and this fact motivated us to improve our system with the capability of incorporating larger training data sets when available.

There are two important modules in this system. The first one uses pattern learning that relies on machine readable dictionaries and sense-tagged corpora to tag all words in open text. The second module is triggered only for words with large training data, as it was the case with the words from the lexical sample tasks. It uses an instance based learning algorithm with automatic feature selection.

To our knowledge, both pattern learning and automatic feature selection are novel approaches in the WSD field, and they led to very good results during the SEnseval-2 evaluation exercise. 


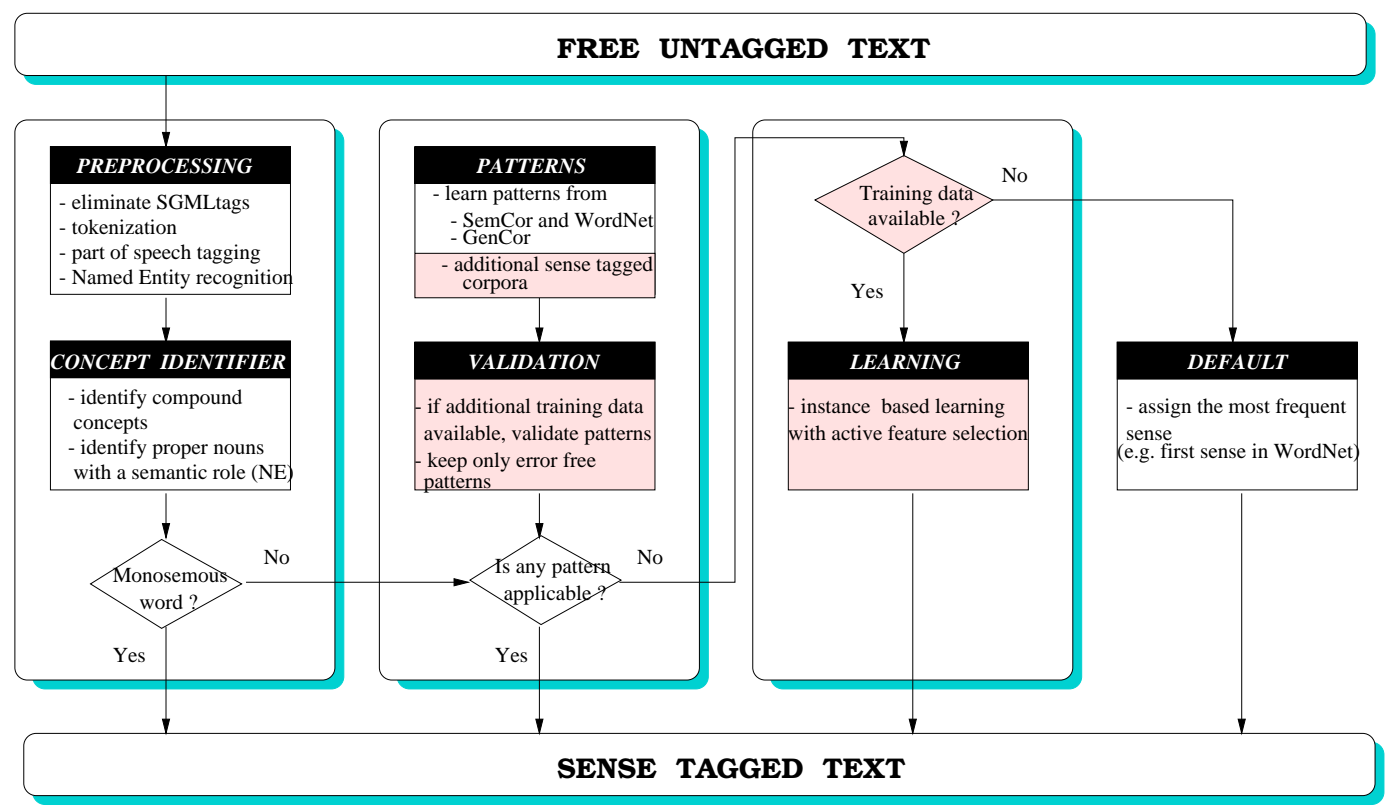

Fig. 1. System architecture

\section{System description}

The WSD algorithm used in this system has the capability of tagging words when no specific sense-tagged corpus is available, automatically scaling up to larger training data when provided. Figure 1 shows the system architecture. There are two main components: (1) pattern learning from available sense-tagged corpora and dictionary definitions and (2) instance based learning with automatic feature selection. The two modules are preceded by a preprocessing phase that includes compound concept identification, followed by a default phase that assigns the most frequent sense as a last resort, when no other previous methods could be applied.

During the preprocessing stage, SGML tags are eliminated, the text is tokenized, part of speech tags are assigned using Brill tagger (Brill1995), and Named Entities (NE) are identified with an in-house implementation of an NE recognizer. To identify collocations, we determine sequences of words that form compound concepts defined in WordNet. There are two possible problems with this approach. The first one concerns subsuming concepts, as in "United States" and "United States of America". In such cases, priority is given to the longest sequence of words. The second possible conflict regards overlapping concepts, like the two different compounds "English Channel" and "Channel Tunnel" found in the text "English Channel Tunnel". Here, we break the tie by keeping the last encountered collocation, with the only reason for this decision being the ease of implementation.

In the second stage, patterns are learned from WordNet, SemCor and GenCor, which is a large sense-tagged corpus automatically built via a set of heuristics (Mihalcea2002). 
If additional training data is available, patterns may be filtered through a validation process. Practically, patterns are applied on the sense-tagged data and they are kept only if no counter-examples are found in the training sets provided.

The third step consists of a learning mechanism with automatic feature selection. This step is initiated only for words with a sufficiently large number of examples, as it was the case with the words in the SENSEVAL lexical sample tasks.

\section{Pattern learning for large vocabulary word sense disambiguation}

Pattern learning and matching is a technique that was successfully used in other NLP tasks, including the disambiguation of confusable word pairs (Brill2000) and shallow parsing (Argamon et al.1998).

Within our system, the pattern learning module is intended for solving the semantic ambiguity of all words in open text. To this end, we build disambiguation patterns using SemCor, WordNet and GenCor. Several processing steps were required to transform the first two resources into a corpus useful for the task of open text WSD. Moreover, these lexical resources coupled with a set of heuristics are used as seeds for generating a new sense-tagged corpus called GenCor.

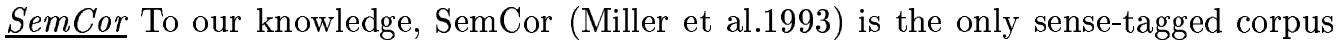
freely available that tags all words in open text. The SENSEVAL-2 English tasks decided to use WordNet 1.7 sense inventory, while SemCor was available only for earlier versions of WordNet. We had therefore to process this corpus and map the WordNet 1.6 senses to their corresponding senses in WordNet 1.7. ${ }^{1}$

WordNet Besides being a large sense inventory, WordNet (Miller1995) can also be used as a source of examples for the different semantic meanings of a word, through the definitions and examples rendered for each word sense. Steps have been taken towards the disambiguation of WordNet glosses (Mihalcea and Moldovan2001), but this project is not yet completed, and therefore we had to rely on shallower methods for identifying word senses within WordNet examples. The main idea in generating a sense-tagged corpus out of WordNet is very simple. It is based on the underlying assumption that each example pertains to a word belonging to the current synset, thereby allowing us to assign the correct sense to at least one word in each example. For instance, the example given for mother\#4 is "necessity is the mother of invention", where the word mother can be tagged with its appropriate sense.

GenCor is a generated sense-tagged corpus. More details on how GenCor is generated are presented in (Mihalcea2002). The algorithm underneath GenCor combines and extends the approaches proposed by (Yarowsky1995) and (Mihalcea and Moldovan1999) in order to obtain large collections of sense-tagged examples. Shortly, the generation algorithm is iterative and consists of three main steps:

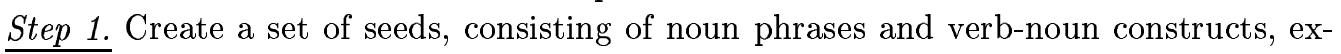
tracted from:

${ }^{1}$ SemCor 1.6 is available for download from the WordNet site http://www.cogsci.princeton.edu/ $\sim$ wn/.

SemCor 1.7 can be downloaded from

http://www.seas.smu.edu/ rada/semcor 


\subsection{SemCor}

1.2 Sense-tagged examples in WordNet

1.3 Sense-tagged examples created with the principles described in (Mihalcea and Moldovan1999). These are examples found on the Web by searching for related unambiguous words. Currently, we only use monosemous synonyms, hypernyms or hyponyms for any given word. For instance, sense-tagged examples for mother\#2 may be obtained by searching for its monosemous hypernym barm, and then replace barm with mother in all the examples that are retrieved.

Step 2. Search the Web for each sequence of words in the seeds set.

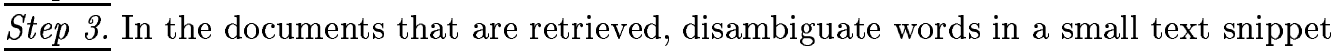
surrounding the searching seed, using the main ideas of the algorithm in (Mihalcea and Moldovan2000). In this algorithm, words are disambiguated based on their relation with other words in their immediate vicinity. To this end, we rely on the synonymy and hypernymy relations, as defined in WordNet. For example, mother and parent found close to each other are disambiguated based on their relation parent\#1 is-hypernym mother\#1. Similarly, car in the immediate vicinity of railcar may be annotated as car\#2, since car\#2 and railcar\#1 are synonyms in WordNet. Noun phrases and verb noun constructs including the newly disambiguated words form new seeds that are added to the seeds set. Go back to step 2 .

Example "blooming plant\#2" is a noun phrase extracted from SemCor as part of the initial set of seeds. A search on the Web for this construct results in several texts, including “(2) Florist item means a cut flower, potted plant, blooming plant, inside foliage plant, bedding plant, corsage flower, cut foliage, floral decoration, or live decorative material. In this text, we disambiguate all instances of plant, and obtain the following new seeds: potted plant\#2, foliage plant\#2, bedding plant\#2. Subsequent searches for these seeds will result in additional texts where new seeds may be extracted. The generation process continues for several iterations, and stops when a certain apriori established number of tagged examples is obtained. For instance, the corpus generated for the English all words task during Senseval-2 consisted of about 160,000 examples.

Once we create this large corpus with examples of word meanings, we can start to extract patterns. For each semantically tagged word found in the corpus, patterns are constructed including the word itself and its local context. The local context is formed with a window of maximum $\mathrm{N}$ words to the left and $\mathrm{M}$ words to the right of the word of interest (currently, $\mathrm{M}=\mathrm{N}=2$ ).

Each word in the corpus is represented by its base form, its part of speech, its sense ${ }^{2}$, if there is any provided, and its hypernym, again if the sense is known. We have therefore the following format for each pattern word: baseform/POS/offset/hypernym-offset. Any of these word components can be unspecified, and therefore denoted with the symbol *. A count is also associated with every pattern, indicating the number of times it occurs in the corpus.

Additionally, a set of constraints is applied to filter out meaningless patterns. For instance, based on the observation that patterns like $<$ the $/ \mathrm{DT} / * / *$ rest $/ \mathrm{NN} / 11411361 / *>$

2 The sense is specified through the synset offset. The benefit of this notation is that we enable synonym matches, e.g. mother\#1 and female_parent\#1 both have the offset 08284239 
(obtained for $\mathrm{N}=1, \mathrm{M}=0$ ) usually lack meaningful information, we filter out all patterns consisting of a DT-NN sequence. These constraints are basically indicators of what word combinations are not allowed in the patterns set. In addition to DT-NN, patterns may not consist of a modal followed by a verb (MD-VB), a noun followed by a conjunction (NN-CC), and others.

When trying to disambiguate a word, we first search for all available patterns that would match the current context. A pattern is said to match the current context if: (1) all words in the pattern are retrieved in the local context in the same order and at the same relative distance with respect to the target word and (2) each pattern word has a complete or partial match with its corresponding context word. A complete match is obtained when all specified word components are matched. If only a subset of the pattern word components find a match among the corresponding context word components, then we have a partial match, and a smaller score is assigned, as shown in the PatternMatching function below. If more than one pattern is available, then the decision of which pattern to apply is based on the pattern strength. The strength of a pattern is evaluated in terms of (1) the number of specified components, (2) the number of occurrences and (3) the length of the pattern. For example, $<$ the/DT/*/* modal/JJ/01551759/* age $/ \mathrm{NN} / *^{*}$ at $/ \mathrm{IN} / *^{*}>$ is considered to be stronger than $<\operatorname{modal} / \mathrm{JJ} / 01551759 / *$ age $/ \mathrm{NN} / * / *>$. Also, <clear/JJ/00406603/* water/NN/12281250/*> is stronger than $<$ clear $/ \mathrm{JJ} / *{ }^{*}$ water $/ \mathrm{NN} / 12281250 /{ }^{*}>$. The hypernym is also provided for the purpose of allowing generalizations. For instance, $<* / \mathrm{NN} / * / 03507584$ door/NN/02746251/*> matches "kitchen door" as well as "bedroom door" (03507584 is the offset for room\#1). The PatternMatching function below illustrates the main steps performed during pattern matching. 


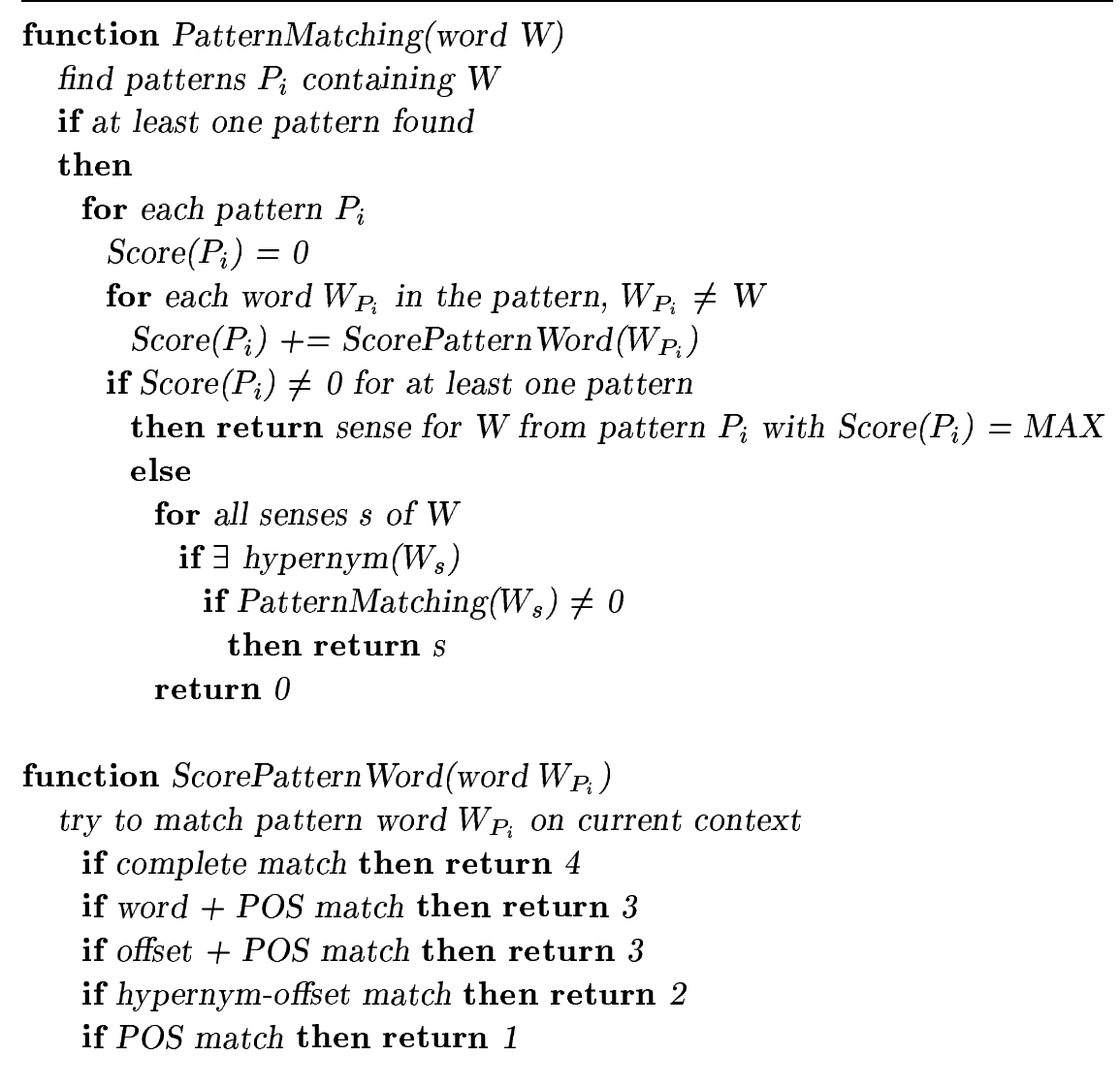

In addition to the all words task, a pattern matching procedure is also performed during the lexical sample task, which includes a validation process where patterns are matched against training data, and only error free patterns are kept.

Another important step performed during the all words disambiguation task is sense propagation. The patterns do not guarantee a complete coverage of all words in input text, and therefore additional methods are required. We use a cache-like procedure that relies on the "one sense per discourse" paradigm to assign to each ambiguous word the sense of its closest occurrence, if any exists. The words left ambiguous at this point are assigned by default the first sense in WordNet.

\section{Learning with automatic feature selection}

Learning mechanisms for disambiguating word sense have a long tradition in the WSD field, including a large range of algorithms and feature types. Most of the efforts in the WSD field have been concentrated so far towards supervised learning algorithms, and these are the methods that achieve the best performance at the cost of low recall (they address only few, pre-selected words). Each sense-tagged occurrence of a particular word is transformed into a feature vector, suitable for an automatic learning process. Two main decisions need to be taken when designing such a system: the set of features to 
be used and the learning algorithm. Commonly used features include surrounding words and their part of speech, context keywords (Ng and Lee1996) or context bigrams (Pedersen2001), and various syntactic properties (Fellbaum et al.2001) etc. As for the learning methodology, a large range of algorithms have been used, including neural networks (Leacock et al.1998), decision trees (Pedersen2001), decision lists (Yarowsky2000), memory based learning (Veenstra et al.2000), and others. An experimental comparison of seven learning algorithms used to disambiguate the meaning of the word line is presented in (Mooney1996). See also (Yarowsky and Florian2002) in this issue.

For our system, we have decided for an instance based algorithm with information gain feature weighting. The reasons for this decision are threefold. First, it has been advocated that forgetting exceptions is harmful for language learning applications (Daelemans et al.1999), and instance based algorithms are known for their property of taking into consideration every single training example when making a classification decision. Secondly, instance based learning algorithms have been successfully used in WSD applications (Veenstra et al.2000). Finally, this type of algorithms is efficient in terms of training and testing time. We initially used the MLC $++{ }^{3}$ implementation, and later on switched to Timbl (Daelemans et al.2001).

Even more important than the choice of learning methodology is the selection of features to be employed during the learning process. Our intuition was that different sets of features have different effects depending on the ambiguous word considered. Usually, features are weighted using weighting schemes that are based on information gain, gain ratio, chi-squared or other information content measures. Still, weights are computed independently for each feature and therefore this strategy does not always guarantee to provide the best results. Sometimes it is better to leave features out than assign them even a small weight (Daelemans et al.2001). We need therefore to identify efficient criteria for feature selection.

Feature selection is a technique that has been successfully used in other Artificial Intelligence applications. (Cardie1996) proposes a linguistic and cognitive biased approach for relative pronoun resolution. In (Aha and Bankert1994)'s system, features are selected using searching algorithms, with increased performance obtained in the problem of cloud types classification. In all these applications, performing feature selection prior to the learning phase was found to be a helpful factor towards increased performance.

For our system, features are automatically selected using a forward search algorithm. The classic approach used so far in WSD was to build word experts via a learning process that determines the values for a pre-selected set of features. Instead, we first learn the set of features that would best model the word characteristics, and therefore exploit at maximum the idiosyncratic nature of words. It is only at a second stage that we actually create the word experts by determining the values for the set of features previously selected.

Using this approach, we combine the advantages of instance based learning mechanisms that have the nice property of "not forgetting exceptions", with an optimized feature selection scheme. One could argue that decision trees have the capability of selecting

\footnotetext{
${ }^{3}$ Machine Learning library available at http://www.sgi.com/tech/mlc
} 
relevant features, but it has been shown (Almuallim and Dietterich1991) that irrelevant features significantly affect the performance of decision trees as well.

The algorithm for automatic feature selection is sketched below.

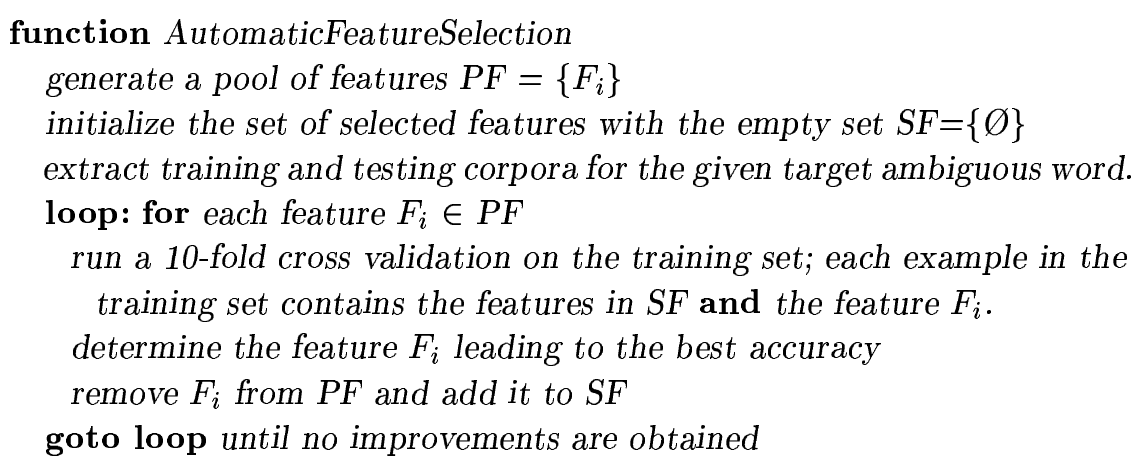

\section{Features that are good indicators of word sense}

Three types of features are distinguished:

1. 0-param features, which can be used or not, without any parameters to set. For example, the part of speech of a surrounding word is a 0-param feature, since a learning example can either contain or omit this feature, without having to indicate a specific parameter.

2. 1-param features, which, once selected, have one variable parameter that can be set to a specific value (alternatively, this parameter may be left with its default value). As an example, consider the context feature $(\mathrm{CF})$, which adds as attributes the words in a surrounding window of length $\mathrm{K}$. Deciding the value for $\mathrm{K}$ implicitly means setting one parameter for this feature.

3. 2-param features with two parameters associated. For example, one can select MX keywords as representative for the context of an ambiguous word, where a keyword is defined as a word occuring at least MN times. Therefore, two parameters have to be set for this feature, MX and MN.

The features that we employed so far are presented below. They form the pool of features $\mathrm{PF}$ from which features are selected using the algorithm described in Section 4. In the following, the ambiguous word is denoted with $A W$.

CW Current word (0-param) The word $A W$ itself, exactly as it occurs in the text. Notation: $C W$

CP Current part of speech (0-param) The part of speech of the word AW. Notation: $C P$

CF Contextual features (1-param) The words and parts of speech of the $\mathrm{K}$ words surrounding $A W$ (Bruce and Wiebe1999). Notation: $C F[=K]$, default=3

COL Collocations (1-param) Collocations formed with maximum $\mathrm{K}$ words surrounding $A W(\mathrm{Ng}$ and Lee1996). Notation: $C O L[=K]$, default $=3$ 
HNP Head of noun phrase 2 (0-param) The head of the noun phrase to which $A W$ belongs, if any. Notation: HNP

SK Sense specific keywords (2-param) Maximum MX keywords occurring at least MN times are determined for each sense of the ambiguous word. The value of this feature is either 0 or 1 , depending whether the current example contains one of the determined keywords or not ( $\mathrm{Ng}$ and Lee1996). Notation: $S K[=M N, M X]$, default=5,5

B Bigrams (2-param) Maximum MX bigrams occurring at least MN times are determined for all training examples. The value of this feature is either 0 or 1 , depending if the current example contains one of the determined bigrams or not. Bigrams are ordered using the Dice coefficient, which gives a measure of association among two words in a corpus. (Pedersen2001) gives several alternatives for measures used in bigrams selection. Notation: $B[=M N, M X]$, default $=5,20$

Other In addition, we have a set of eleven other features that refer to surrounding words with a given part of speech: Verb before (VB 0-param), Verb after (VA 0-param), Noun before (NB 0-param), Noun after (NA 0-param), Named Entity before (NEB 0-param), Named Entity after (NEA 0-param), Preposition before (PB 0-param), Preposition after (PA 0-param), Pronoun before (PRB 0-param), Pronoun after (PRA 0-param), Determiner before (DT 0-param).

New features can be easily added to the pool, with no changes required in the main algorithm. We have initially tested the system on the Senseval-1 data, in an noncompetitional environment, when there was enough time to parse the data. Two additional features were considered at that time to help towards performance. We decided not to use them in the current experiments, mainly for time considerations, since parsing is a highly computationally intensive task.

PPT Parse path (1-param) Maximum K parsing labels found on the path to the top of the parse tree (sentence top). Notation: PPT $[=K]$, default=10. For instance, given the parse tree (S (NP (JJ big) (NN house))), the value for this feature for the noun house is $N N, N P, S$.

SPC Same parse phrase components (1-param) Maximum K parse components found in the same phrase as $A W$. Notation: $S P C[=K]$, default=3. For the example above, this feature would be set to $J J, N N$.

\section{Results on Senseval-2 data}

The overall performance of the system on the provided during the English all words task was $69 \%$ for fine-grained scoring, and $69.8 \%$ for coarse-grained scoring. On the English lexical sample data, we obtained $63.8 \%$ for fine-grained scoring, and $71.2 \%$ for coarsegrained scoring. These results rank this system as the best performing one in the ranking made before the deadline. See (Edmonds and Cotton2002) for details on SEnseval-2.

Tables 1,2 and 3 present the results obtained during the lexical sample task, for 73 ambiguous words, including 29 nouns, 15 adjectives and 29 verbs. For each word, the table shows: the number of examples in the training and test sets; the features automatically selected as a result of applying the algorithm in Section 4; the 10-fold cross validation precision obtained on training data with the selected features set; the 
precision for fine-grained and coarse-grained scoring as computed by the SENSEVAL-2 organizers. Collocations are identified since the preprocessing stage and the learning process is applied separately on each concept ${ }^{4}$, but due to space limitations, the table shows only features and results obtained for single words.

\begin{tabular}{|c|c|c|c|c|c|c|}
\hline \multirow[b]{2}{*}{ word.pos } & \multicolumn{2}{|c|}{ Size } & \multirow[b]{2}{*}{ Features } & \multirow{2}{*}{$\begin{array}{l}10 \text {-fold } \\
\text { valid. }\end{array}$} & \multicolumn{2}{|c|}{ SENSEVAL score } \\
\hline & train & test & & & fine | & coarse \\
\hline art.n & 194 & 98 & $\mathrm{CF}=1 \mathrm{HNP} \mathrm{B}=2,5 \mathrm{VB} \mathrm{NB}$ & $60.6 \%$ & $71.4 \%$ & $74.5 \%$ \\
\hline authority.n & 183 & 92 & $\mathrm{CW} \mathrm{CP} \mathrm{COL}=1 \mathrm{VB} \mathrm{NB}$ & $62.2 \%$ & $70.7 \%$ & $91.3 \%$ \\
\hline bar.n & 264 & 151 & $\mathrm{CW} C \mathrm{CP} C \mathrm{CO}=1 \mathrm{COL}=1 \mathrm{~B}=5,3 \mathrm{VB} \mathrm{NB} \mathrm{NEA}$ & $60.8 \%$ & $62.3 \%$ & $74.5 \%$ \\
\hline bum.n & 80 & 45 & CW NA NEA & $86.2 \%$ & $77.8 \%$ & $80.0 \%$ \\
\hline chair.n & 137 & 69 & $\mathrm{CW}$ & $92.3 \%$ & $85.5 \%$ & $88.4 \%$ \\
\hline channel.n & 138 & 73 & CP NB & $43.0 \%$ & $46.6 \%$ & $56.2 \%$ \\
\hline child.n & 129 & 64 & $\mathrm{CW}$ CP $\mathrm{CF}=1 \mathrm{COL}=1 \mathrm{~B}=5,3 \mathrm{NB}$ NEB DT & $76.1 \%$ & $68.8 \%$ & $68.8 \%$ \\
\hline church.n & 128 & 64 & $\mathrm{CW} \mathrm{CP} \mathrm{CF}=2 \mathrm{COL}=1 \mathrm{~B}=5,1$ & $64.4 \%$ & $56.2 \%$ & $56.2 \%$ \\
\hline circuit.n & 169 & 85 & $\mathrm{CP} \mathrm{CF}=3 \mathrm{~B}=5,1 \mathrm{VB}$ & $51.6 \%$ & $58.8 \%$ & $62.4 \%$ \\
\hline day.n & 289 & 145 & $\mathrm{CP} \mathrm{CF}=2 \mathrm{HNP} \mathrm{NEB} \mathrm{PB}$ & $78.0 \%$ & $76.1 \%$ & $77.3 \%$ \\
\hline detention.n & 63 & 32 & any & $94.0 \%$ & $87.5 \%$ & $87.5 \%$ \\
\hline dyke.n & 58 & 28 & $\mathrm{CW} \mathrm{CF}=2 \mathrm{SK}=5,2$ & $91.4 \%$ & $89.3 \%$ & $89.3 \%$ \\
\hline facility.n & 114 & 58 & $\mathrm{CP} C O L=1 \mathrm{VB} P R B$ & $74.5 \%$ & $79.3 \%$ & $98.3 \%$ \\
\hline fatigue.n & 76 & 43 & $\mathrm{CP} \mathrm{B}=5,3 \mathrm{NB}$ & $86.6 \%$ & $88.4 \%$ & $90.7 \%$ \\
\hline feeling.n & 102 & 51 & $\mathrm{CP} C F=1 \mathrm{COL}=3 \mathrm{HNP}$ NEA & $64.0 \%$ & $74.5 \%$ & $74.5 \%$ \\
\hline grip.n & 100 & 51 & $\mathrm{CP} \mathrm{CF}=3 \mathrm{COL}=2 \mathrm{~PB} \mathrm{DT}$ & $60.0 \%$ & $41.2 \%$ & $58.8 \%$ \\
\hline hearth.n & 64 & 32 & $\mathrm{CP} \mathrm{CF}=1 \mathrm{HNP}$ & $66.7 \%$ & $75.0 \%$ & $87.5 \%$ \\
\hline holiday.n & 62 & 31 & $\mathrm{CP}$ & $96.0 \%$ & $93.5 \%$ & $96.8 \%$ \\
\hline lady.n & 103 & 53 & CW HNP & $84.0 \%$ & $88.7 \%$ & $94.3 \%$ \\
\hline material.n & 140 & 69 & $\mathrm{CW} C \mathrm{CP} \mathrm{COL}=1 \mathrm{~B}=2,5$ VA NEA & $53.3 \%$ & $56.5 \%$ & $60.9 \%$ \\
\hline mouth.n & 118 & 60 & $\mathrm{CP} \mathrm{COL}=1 \mathrm{VB} \mathrm{NB} \mathrm{PB}$ & $65.7 \%$ & $65.0 \%$ & $93.3 \%$ \\
\hline nation.n & 75 & 37 & $\mathrm{CP}$ & $80.0 \%$ & $54.1 \%$ & $54.1 \%$ \\
\hline nature.n & 92 & 46 & CP DT & $58.0 \%$ & $69.6 \%$ & $80.4 \%$ \\
\hline post.n & 150 & 79 & $\mathrm{CW} \mathrm{CP} \mathrm{CF}=1 \mathrm{COL}=2$ & $74.6 \%$ & $64.6 \%$ & $68.4 \%$ \\
\hline restraint.n & 91 & 45 & $\mathrm{CP} \mathrm{COL}=2 \mathrm{HNP} \mathrm{B}=2,5 \mathrm{VB} \mathrm{NB} \mathrm{PA}$ & $67.3 \%$ & $62.2 \%$ & $71.1 \%$ \\
\hline sense.n & 107 & 53 & $\mathrm{CP} \mathrm{CF}=1 \mathrm{~B}=3,3 \mathrm{NEB} \mathrm{PB}$ & $74.5 \%$ & $75.5 \%$ & $74.4 \%$ \\
\hline spade.n & 64 & 33 & $\mathrm{CP} \mathrm{CF}=1 \mathrm{COL}=2$ & $94.0 \%$ & $97.0 \%$ & $97.0 \%$ \\
\hline stress.n & 78 & 39 & $\mathrm{CP} \mathrm{COL}=2 \mathrm{~B}=5,2$ & $68.0 \%$ & $64.1 \%$ & $89.7 \%$ \\
\hline yew.n & 57 & 28 & $\mathrm{CF}=1$ & $94.0 \%$ & $89.3 \%$ & $100.0 \%$ \\
\hline TOTAL.N & 3,523 & 1,759 & - & - & $69.5 \%$ & $76.6 \%$ \\
\hline
\end{tabular}

Table 1. Training and test sizes, optimal feature sets and precisions (10-fold on training data, fine-grained and coarse-grained on test data) for 29 nouns

For the 1-param and 2-param features, there is a range of values allowed for their parameters: [1-5] for the 1-param features, and [1-10] for the 2-param features. This means that, for instance, $\mathrm{CF}$ can be set to $\mathrm{CF}=1, \mathrm{CF}=2, \mathrm{CF}=3, \mathrm{CF}=4$ or $\mathrm{CF}=5$. The selection of the best value is performed empirically using the same cross-validation algorithm.

When no training data is provided (as was the case with the SENSEVAL-2 verb "keep going"), the first sense is applied by default. Also, when the training set size is smaller than 15 examples, we do not use the automatic feature selection algorithm; we use instead a default set of features ( $\mathrm{CW} \mathrm{CP} \mathrm{CF}=1 \mathrm{COL}=1)$.

4 Training and testing corpora are extracted for each ambiguous word. This means that examples pertaining to the multiword "dress down" are separated from the examples for the single word "dress" 


\begin{tabular}{|c|c|c|c|c|c|c|}
\hline \multirow[b]{2}{*}{ word.pos } & \multicolumn{2}{|c|}{ Size } & \multirow[b]{2}{*}{ Features } & \multirow{2}{*}{$\begin{array}{l}\text { 10-fold } \\
\text { valid. }\end{array}$} & \multicolumn{2}{|c|}{ SEnSEVAL score } \\
\hline & train & test & & & fine | & coarse \\
\hline blind.a & 105 & 55 & $\mathrm{HNP}$ & $70.0 \%$ & $85.5 \%$ & $85.5 \%$ \\
\hline colourless.a & 68 & 35 & $\mathrm{CW} \mathrm{CP} \mathrm{CF}=1 \mathrm{COL}=1 \mathrm{SK}=3,3$ & $85.7 \%$ & $48.6 \%$ & $48.6 \%$ \\
\hline cool.a & 103 & 52 & $\mathrm{CF}=1 \mathrm{COL}=2 \mathrm{HNP} \mathrm{VB}$ PB PRB DT & $56.1 \%$ & $51.9 \%$ & $51.9 \%$ \\
\hline faithful.a & 47 & 23 & $\mathrm{CW}$ & $68.0 \%$ & $87.0 \%$ & $87.0 \%$ \\
\hline fine.a & 139 & 70 & $\mathrm{CP} \mathrm{CF}=2 \mathrm{HNP} \mathrm{B}=5,1 \mathrm{NA}$ & $46.0 \%$ & $54.3 \%$ & $54.3 \%$ \\
\hline fit.a & 57 & 29 & $\mathrm{CF}=1 \mathrm{~B}=3,3 \mathrm{VB} \mathrm{NA}$ & $85.0 \%$ & $82.8 \%$ & $82.8 \%$ \\
\hline free.a & 165 & 82 & $\mathrm{CP} \mathrm{CF}=1 \mathrm{COL}=2$ & $65.0 \%$ & $58.5 \%$ & $58.5 \%$ \\
\hline graceful.a & 56 & 29 & $\mathrm{CW}$ & $87.0 \%$ & $79.3 \%$ & $79.3 \%$ \\
\hline green.a & 190 & 94 & CP VA & $80.0 \%$ & $79.8 \%$ & $79.8 \%$ \\
\hline local.a & 75 & 38 & CP NA & $88.0 \%$ & $81.6 \%$ & $81.6 \%$ \\
\hline natural.a & 205 & 103 & CP $C F=1$ HNP VB NB NEB PRA & $50.0 \%$ & $56.3 \%$ & $56.3 \%$ \\
\hline oblique.a & 56 & 29 & $\mathrm{CW} C \mathrm{CP} \mathrm{CF}=1 \mathrm{COL}=4 \mathrm{~B}=3,3$ & $84.0 \%$ & $86.2 \%$ & $86.2 \%$ \\
\hline simple.a & 130 & 66 & $\mathrm{CP} C F=1 \mathrm{COL}=2 \mathrm{HNP}$ NA PB PRA DT & $53.3 \%$ & $53.0 \%$ & $53.0 \%$ \\
\hline solemn.a & 52 & 25 & $\mathrm{CP} \mathrm{COL}=1 \mathrm{DT}$ & $92.8 \%$ & $96.0 \%$ & $96.0 \%$ \\
\hline vital.a & 74 & 38 & CW CP NB & $88.7 \%$ & $94.7 \%$ & $94.7 \%$ \\
\hline TOTAL.A & 1,535 & 768 & - & - & $68.8 \%$ & $68.8 \%$ \\
\hline
\end{tabular}

Table 2. Training and test sizes, optimal feature sets and precisions (10-fold on training data, fine-grained and coarse-grained on test data) for 15 adjectives

\subsection{Discussion}

The all words task owes its performance to SemCor, WordNet, GenCor, the pattern learning procedure, the cache-like sense propagation algorithm and the simple "most frequent sense" heuristic. We address all open class words in open text, and therefore a recall of $100 \%$ is obtained on this data. From this, a coverage of $40.23 \%$ is due to pattern learning, $7.84 \%$ to sense propagation, and the rest of $51.93 \%$ is attained by tagging words with their most frequent sense. If only the last procedure is applied on the entire data set, the overall precision drops to $63.89 \%$, which may be considered as a baseline for this task.

To determine the contributuion of the various knowledge sources, and find the raise in precision brought by the use of GenCor, we performed two comparative experiments: one where only SemCor and WordNet were employed as sources of tagged data, and a second one where GenCor was used in addition to these two resources. The overall precision obtained during the first experiment was $65.1 \%$, while the second experiment led to a precision of $69.3 \%$, therefore more than $4 \%$ precision are gained due to GenCor.

The disambiguation of the words in the lexical sample task relies mainly on the SENSEVAL training data and the instance based learning algorithm with automatic feature selection, which provides complete coverage of the test data. Table 4 lists the number of times each feature was used in the semantic disambiguation of nouns, verbs and adjectives. The most often used features turn out to be $\mathrm{CW}, \mathrm{CP}, \mathrm{CF}$ and $\mathrm{COL}$, which are also the features most frequently mentioned in the literature. Almost all words took advantage of the current part of speech (CP) feature. This is in agreement with (Stevenson and Wilks2001), who have emphasized the major role played by part of speech in WSD. It is interesting to observe that in terms of words in context, bigrams seem to be more 


\begin{tabular}{|c|c|c|c|c|c|c|}
\hline \multirow[b]{2}{*}{ word.pos } & \multicolumn{2}{|c|}{ Size } & \multirow[b]{2}{*}{ Features } & \multirow{2}{*}{$\begin{array}{l}10 \text {-fold } \\
\text { valid. }\end{array}$} & \multicolumn{2}{|c|}{ SEnSEVAL score } \\
\hline & train & test & & & fine $\mid$ & coarse \\
\hline begin. $v$ & 557 & 280 & $\mathrm{CF}=1 \mathrm{NA}$ & $80.40 \%$ & $87.5 \%$ & $87.5 \%$ \\
\hline call.v & 132 & 66 & $\mathrm{CF}=1 \mathrm{COL}=2 \mathrm{VB} \mathrm{NB} \mathrm{DT}$ & $70.00 \%$ & $40.9 \%$ & $66.7 \%$ \\
\hline carry.v & 132 & 66 & $\mathrm{CW} \mathrm{CP} \mathrm{COL}=1 \mathrm{NB}$ & $35.00 \%$ & $39.4 \%$ & $50.0 \%$ \\
\hline collaborate.v & 57 & 30 & $\mathrm{CW} \mathrm{CP} \mathrm{CF}=1$ & $95.80 \%$ & $90.0 \%$ & $90.0 \%$ \\
\hline develop.v & 133 & 69 & $\mathrm{CW} C \mathrm{CP} B=2,5 \mathrm{NA} \mathrm{PB}$ & $22.50 \%$ & $36.2 \%$ & $49.3 \%$ \\
\hline draw.v & 82 & 41 & $\mathrm{CF}=2 \mathrm{COL}=2 \mathrm{NEB}$ & $11.00 \%$ & $31.7 \%$ & $43.9 \%$ \\
\hline dress.v & 119 & 59 & $\mathrm{CP} C F=1 \mathrm{NB} N A \mathrm{~PB}$ & $57.50 \%$ & $57.6 \%$ & $86.4 \%$ \\
\hline drift.v & 63 & 32 & $\mathrm{CW} C \mathrm{CP} \mathrm{CF}=2 \mathrm{COL}=3 \mathrm{HNP}$ NEB PA & $22.00 \%$ & $59.4 \%$ & $62.5 \%$ \\
\hline drive.v & 84 & 42 & $\mathrm{CW} C \mathrm{CP} C \mathrm{C}=2 \mathrm{PRA} \mathrm{DT}$ & $45.00 \%$ & $52.4 \%$ & $69.0 \%$ \\
\hline face.v & 186 & 93 & $\mathrm{CP}$ & $84.00 \%$ & $81.7 \%$ & $90.3 \%$ \\
\hline ferret.v & 2 & 1 & any & - & $100.0 \%$ & $100.0 \%$ \\
\hline find.v & 132 & 68 & $\mathrm{CP} \mathrm{CF}=2 \mathrm{SK}=5,2$ & $10.00 \%$ & $29.4 \%$ & $39.7 \%$ \\
\hline keep.v & 133 & 67 & $\mathrm{CP} \mathrm{B}=3,3$ & $38.00 \%$ & $44.8 \%$ & $46.3 \%$ \\
\hline leave.v & 132 & 66 & $\mathrm{CP} C F=1 \mathrm{COL}=3 \mathrm{NEA}$ & $28.90 \%$ & $47.0 \%$ & $53.0 \%$ \\
\hline live.v & 129 & 67 & CP NA & $63.00 \%$ & $67.2 \%$ & $68.7 \%$ \\
\hline match.v & 86 & 42 & CW CP HNP SK $=5,5$ NA & $26.40 \%$ & $40.5 \%$ & $59.5 \%$ \\
\hline play.v & 129 & 66 & $\mathrm{CW} \mathrm{CP} \mathrm{CF}=4 \mathrm{COL}=4 \mathrm{VB} \mathrm{NA}$ & $21.00 \%$ & $50.0 \%$ & $51.5 \%$ \\
\hline pull.v & 122 & 60 & $\mathrm{CP} \mathrm{COL}=1 \mathrm{HNP} \mathrm{B}=2,10 \mathrm{SK}=5,5$ & $23.00 \%$ & $48.3 \%$ & $68.3 \%$ \\
\hline replace.v & 86 & 45 & $\mathrm{CP} \mathrm{COL}=3 \mathrm{SK}=5,1 \quad \mathrm{~B}=3,2$ & $54.00 \%$ & $44.4 \%$ & $88.9 \%$ \\
\hline see.v & 131 & 69 & $\mathrm{CW} \mathrm{CP} \mathrm{CF}=2 \mathrm{SK}=4,4 \mathrm{~PB}$ & $23.00 \%$ & $37.7 \%$ & $42.0 \%$ \\
\hline serve.v & 100 & 51 & $\mathrm{CP} \mathrm{CF}=4 \mathrm{HNP} \mathrm{B}=5,5$ VA NEB PRB PRA & $36.00 \%$ & $49.0 \%$ & $54.9 \%$ \\
\hline strike.v & 104 & 54 & $\mathrm{CW} C \mathrm{CP} \mathrm{CF}=2 \mathrm{NEB}$ & $23.00 \%$ & $38.9 \%$ & $51.9 \%$ \\
\hline train.v & 125 & 63 & $\mathrm{CW} C \mathrm{CP}=2 \mathrm{COL}=4 \mathrm{NA} \mathrm{PB} \mathrm{PA} \mathrm{DT}$ & $34.00 \%$ & $41.3 \%$ & $52.4 \%$ \\
\hline treat.v & 88 & 44 & $\mathrm{CP} C F=3 \mathrm{COL}=2 \mathrm{VB}$ NEA PA PRB PRA & $36.00 \%$ & $63.6 \%$ & $79.5 \%$ \\
\hline turn.v & 131 & 67 & $\mathrm{CP} \mathrm{CF}=2 \mathrm{VB}$ NA PA PRB & $30.70 \%$ & $35.8 \%$ & $53.7 \%$ \\
\hline use.v & 147 & 76 & CW CP NA VA PRB & $65.00 \%$ & $72.4 \%$ & $84.2 \%$ \\
\hline wander.v & 100 & 50 & CP PA & $81.00 \%$ & $74.0 \%$ & $90.0 \%$ \\
\hline wash.v & 25 & 12 & $\mathrm{CW} \mathrm{CP} \mathrm{CF}=2 \mathrm{COL}=2 \mathrm{SK}=3,5 \mathrm{NEA}$ & $32.00 \%$ & $66.7 \%$ & $83.3 \%$ \\
\hline work.v & 119 & 60 & $\mathrm{CW} C \mathrm{CP} \mathrm{CF}=2 \mathrm{COL}=2 \mathrm{~B}=3,3 \mathrm{NA} \mathrm{PA}$ & $42.00 \%$ & $43.3 \%$ & $58.3 \%$ \\
\hline TOTAL.V & 3,673 & 1,857 & - & - & $56.4 \%$ & $67.0 \%$ \\
\hline
\end{tabular}

Table 3. Training and test sizes, optimal feature sets and precisions (10-fold on training data, fine-grained and coarse-grained on test data) for 29 verbs

effective than simple keywords. Also, the best setting for the CF feature was found to be a one or two word window.

In terms of average number of features, the semantic disambiguation of nouns requires the smallest number of features (3.7), followed by adjectives (4.4) and verbs (4.5). These statistics are not yet conclusive, since they are computed for a small number of words, but they are indicative of the complexity of the task for various parts of speech. Further investigations and larger amounts of data will eventually confirm this preliminary conclusion.

Several interesting cases were encountered in the SEnseval-2 data, justifying our approach of using automatic feature selection. The influence of a feature greatly depends on the target word: a feature can increase the precision for a word, while making things worse for another word. For instance, a word such as free does not benefit from the SK feature, whereas colourless gains almost $7 \%$ in precision when this feature is used. 


\begin{tabular}{l|c|c|c|c}
\hline & \multicolumn{3}{|c|}{ Part of speech } & \multirow{2}{*}{ Total } \\
\cline { 2 - 4 } & Noun & Verb & Adjective & 73 \\
\hline Words & 29 & 29 & 15 & \\
Features & & & & 32 \\
CW & 10 & 13 & 9 & 61 \\
CP & 22 & 25 & 14 & 40 \\
CF & 14 & 18 & 8 & 31 \\
COL & 13 & 12 & 6 & 15 \\
HNP & 6 & 4 & 5 & 10 \\
SK & 1 & 6 & 3 & 19 \\
B & 10 & 6 & 3 & 14 \\
VB & 7 & 4 & 3 & 4 \\
VA & 1 & 2 & 1 & 13 \\
NB & 8 & 3 & 2 & 15 \\
NA & 1 & 10 & 4 & 8 \\
NEB & 3 & 4 & 1 & 7 \\
NEA & 4 & 3 & 0 & 10 \\
PB & 4 & 4 & 2 & 7 \\
PA & 1 & 6 & 0 & 6 \\
PRB & 1 & 4 & 1 & 5 \\
PRA & 0 & 3 & 2 & 9 \\
DT & 3 & 3 & 3 & 67 \\
Total & 109 & 130 & & 306 \\
\hline
\end{tabular}

Table 4. Feature distribution for nouns, verbs, adjectives

$\begin{array}{lll}\text { free. } a[\mathrm{CW} \mathrm{CP} \mathrm{CF}=1 \mathrm{SK}=3,3] & \rightarrow & 57.85 \% \\ \text { free. } a[\mathrm{CW} \mathrm{CP} \mathrm{CF}=1] & \rightarrow & 63.57 \% \\ \text { colorless. } a[\mathrm{CW} \mathrm{CP} \mathrm{CF}=1] & \rightarrow & 78.57 \% \\ \text { colorless. } a[\mathrm{CW} \mathrm{CP} \mathrm{CF}=1 \mathrm{SK}=3,3] & \rightarrow & 85.71 \%\end{array}$

Another interesting example is the noun chair, which was disambiguated with high precision by simply using the current word (CW) feature. This is explained by the fact that the most frequent senses are Chair meaning person and chair meaning furniture, and therefore the distinction between lower and upper case spellings makes the distinction among the different meanings of this word.

The noun detention has the same precision computed during the 10 -fold cross validation runs, independent of the feature or combination of features used. This is because of the two senses it has, one of them occurs in $97 \%$ of the examples, and hence it statistically dominates the other sense. There were several other interesting cases, including the adjective local which gained $20 \%$ in precision by simply using the feature NA, the word faithful which is best disambiguated with the $\mathrm{CW}$ feature, and others.

The system was also tested on the SEnseval-1 data (Kilgarriff and Palmer2000), where the disambiguation task was performed with respect to Hector dictionary. The overall result achieved on this data was higher than the one reported by the best performing system. Besides proving the validity of our approach, this fact also proved that our system is not tight in any ways to the sense inventory or data format employed. Going from Senseval-1 to Senseval-2 required only minimal changes in the system, mainly in the preprocessing phase (to accept as input the new data format) and in the postprocessing phase (to output the answer sense keys in the format required). 


\section{Conclusion}

Pattern learning and automatic feature selection are new approaches in the WSD field. They have been implemented in a system that was evaluated on the SEnsEval-2 data, with an excellent performance in both English all words and English lexical sample tasks.

Patterns represent a great way of capturing contexts representative for a word meaning. The usage of hypernyms as one of the pattern components gives us the means for generalization beyond words explicitly expressed in text.

In supervised learning algorithms, instance based learning with feature weighting provides a performance comparable with the best results achieved so far in word sense disambiguation. Its performance is greatly increased if coupled with an algorithm for automatic feature selection. This process is completely automated and it practically creates a classifier tailored to the behaviour of each ambiguous word.

\section{Acknowledgments}

The author would like to thank the anonymous reviewers for their feedback and useful comments, which were very helpful in preparing the final version of this paper.

\section{References}

D.W. Aha and R.L. Bankert. 1994. Feature selection for case-based classification of cloud types: An empirical comparison. In Proceedings of the AAAI'94 Workshop on CaseBased Reasoning, pages 106-112, Seattle, WA.

H. Almuallim and T.G. Dietterich. 1991. Learning with many irrelevant features. In Proceedings of the Ninth National Conference on Artificial Intelligence (AAAI-91), volume 2, pages 547552, Anaheim, California.

S. Argamon, I. Dagan, and Y. Krymolowski. 1998. A memory-based approach to learning shallow natural language patterns. In Proceedings of the 17th International Conference on Computational Linguistics (COLING-ACL-98), Montreal, Canada.

E. Brill. 1995. Transformation-based error driven learning and natural language processing: A case study in part-of-speech tagging. Computational Linguistics, 21(4):543-566, December.

E. Brill. 2000. Pattern-based disambiguation for natural language processing. In Proceedings of the Conference on Empirical Methods in Natural Language Processing EMNLP, Hong Kong.

R. Bruce and J. Wiebe. 1999. Decomposable modeling in natural language processing. Computational Linguistics, 25(2):195-207.

C. Cardie. 1996. Automating feature set selection for case-based learning of linguistic knowledge. In Proceedings of the Conference on Empirical Methods in Natural Language Processing $E M N L P$, pages 113-126, Somerset, New Jersey.

W. Daelemans, A. van den Bosch, and J. Zavrel. 1999. Forgetting exceptions is harmful in language learning. Machine Learning, 34(1-3):11-34.

W. Daelemans, J. Zavrel, K. van der Sloot, and A. van den Bosch. 2001. Timbl: Tilburg memory based learner, version 4.0, reference guide. Technical report, University of Antwerp.

P. Edmonds and S. Cotton. 2002. Senseval-2: Overview. In Proceedings of Senseval-2 Workshop, Association of Computational Linguistics, pages 1-6, Toulouse, France.

C. Fellbaum, M. Palmer, H.T. Dang, L. Delfs, and S. Wolf. 2001. Manual and automatic semantic annotation with WordNet. In WordNet and Other lexical resources: NAACL 2001 workshop, pages 3-10, Pittsburgh, June.

A. Kilgarriff and M. Palmer, editors. 2000. Computer and the Humanities. Special issue: SENSEVAL. Evaluating Word Sense Disambiguation programs, volume 34, April.

C. Leacock, M. Chodorow, and G.A. Miller. 1998. Using corpus statistics and WordNet relations for sense identification. Computational Linguistics, 24(1):147-165. 
R. Mihalcea and D.I. Moldovan. 1999. An automatic method for generating sense tagged corpora. In Proceedings of AAAI-99, pages 461-466, Orlando, FL, July.

R. Mihalcea and D.I. Moldovan. 2000. An iterative approach to word sense disambiguation. In Proceedings of FLAIRS-2000, pages 219-223, Orlando, FL, May.

R. Mihalcea and D. Moldovan. 2001. eXtended WordNet: progress report. In NAACL 2001 Workshop on WordNet and Other Lexical Resources: applications, extensions and customizations, pages 95-100, Pittsburgh, June.

R. Mihalcea. 2002. Bootstrapping large sense tagged corpora. In Proceedings of the Third International Conference on Language Resources and Evaluation LREC 2002, pages 14071411, Canary Islands, Spain, May.

G. Miller, C. Leacock, T. Randee, and R. Bunker. 1993. A semantic concordance. In Proceedings of the 3rd DARPA Workshop on Human Language Technology, pages 303-308, Plainsboro, New Jersey.

G. Miller. 1995. Wordnet: A lexical database. Communication of the ACM, 38(11):39-41.

R. Mooney. 1996. Comparative experiments on disambiguating word senses: An illustration of the role of bias in machine learning. In Proceedings of the 1996 Conference on Empirical Methods in Natural Language Processing (EMNLP-1996), pages 82-91, Philadelphia, May.

H.T. Ng and H.B. Lee. 1996. Integrating multiple knowledge sources to disambiguate word sense: An examplar-based approach. In Proceedings of the 34th Annual Meeting of the Association for Computational Linguistics (ACL-96), Santa Cruz.

T. Pedersen. 2001. A decision tree of bigrams is an accurate predictor of word sense. In Proceedings of the North American Chapter of the Association for Compuatational Linguistics, NAACL 2001, pages 79-86, Pittsburg, June.

M. Stevenson and Y. Wilks. 2001. The interaction of knowledge sources in word sense disambiguation. Computational Linguistics, 27(3):321-351.

J. Veenstra, A. van den Bosch, S. Buchholz, W. Daelemans, and J. Zavrel. 2000. Memory-based word sense disambiguation. Computers and the Humanities, 34:171-177.

D. Yarowsky and R. Florian. 2002. Evaluating sense disambiguation across diverse parameter spaces. JNLE Special Issue on Evaluating Word Sense Disambiguation Systems. (this issue).

D. Yarowsky. 1995. Unsupervised word sense disambiguation rivaling supervised methods. In Proceedings of the 33rd Annual Meeting of the Association for Computational Linguistics (ACL-95), pages 189-196, Cambridge, MA, 1995.

D. Yarowsky. 2000. Hierarchical decision lists for word sense disambiguation. Computers and the Humanities, 34:179-186. 